\title{
Principais Características do Consumo de Duráveis no Brasil e Testes de Separabilidade entre Duráveis e Não-Duráveis*
}

\author{
Fábio Augusto Reis Gomes ${ }^{* *}$ \\ João Victor Issler ${ }^{* * *}$ \\ Márcio Antônio Salvato ${ }^{* * * *}$
}

Sumário: 1. Introdução; 2. Teoria econômica e restrições testáveis; 3. Base de dados e análise preliminar; 4. Estimações e resultados; 5. Conclusões.

Palavras-chave: consumo de duráveis; separabilidade; teoria da renda permanente; restrição de crédito.

Códigos JEL: C22; E21.

Este artigo investiga amplamente a evolução do consumo de bens duráveis no Brasil a partir da decisão de consumo individual e da possibilidade de existir restrição ao crédito. A contribuição mais relevante consiste na não rejeição da hipótese de separabilidade nas decisões de consumo de bens duráveis e não-duráveis, já que tal hipótese é implicitamente utilizada por vários artigos que trataram a questão do consumo agregado no Brasil. Os resultados, aqui encontrados, sugerem que uma grande parcela dos consumidores está restrita ao crédito, existindo restrições de curto prazo e longo prazo sobre a evolução do consumo de bens duráveis, não-duráveis e renda.

This paper studies the evolution of the consumption of durables in Brazil and tests the hypothesis of liquidity-constraints for the optimal decision of the representative agent. We also test whether consumption decisions on durable and non-durables are separable, which is critical in understanding consumption behavior and is assumed rather than tested in all previous papers investigating consumption in Brazil. Our empirical results suggest that a large pro-

\footnotetext{
*Artigo recebido em dez. 2003 e aprovado em jun. 2004. Este projeto teve o financiamento do Ministério da Fazenda, do PRONEX, do CNPq, da CAPES e da FAPERJ. Agradecemos as críticas e sugestões de Ricardo Cavalcanti, Marcelo Fernandes (Editor) e de dois pareceristas anônimos, isentando-os da responsabilidade por quaisquer erros aqui remanescentes. Agradecemos ainda a Roberto Ellery por disponibilizar parte da base de dados.

${ }^{* *}$ Escola de Governo da Fundação João Pinheiro e CEPE. E-mail: fabio@cepe.ecn.br

*** EPGE/FGV. E-mail: jissler@fgv.br

${ }^{* * * *}$ PUC Minas e CEPE. E-mail: salvato@pucminas.br
} 
portion of consumers in Brazil face short-run liquidity constraints in the consumption of durables. We also find short-term restrictions involving the behavior of the consumption of non-durable goods, durable goods, and real income.

\section{Introdução}

No Brasil, como na maioria dos países, o consumo agregado é uma das séries macroeconômicas de maior importância, pois, além de representar mais do que $70 \%$ do Produto Interno Bruto, é um argumento fundamental na função de bemestar econômico. A não ser que a função utilidade seja separável, estudos sobre o consumo deveriam tratar de forma integrada as decisões sobre diferentes tipos de gasto. Em particular as decisões sobre o consumo de duráveis e de não-duráveis deveriam ser modeladas de forma integrada, possivelmente incluindo também a renda do consumidor nesta análise. Infelizmente, estudos anteriores sobre o consumo no Brasil não o fizeram, pois consideraram apenas o consumo total, que agrega essas duas categorias - ver Cavalcanti (1993), Reis et alii (1998), Issler e Rocha (2000) e Gomes (2004) - sendo importante que se teste a separabilidade da função utilidade para que se cogite validar alguns desses estudos prévios. Como nota Bernanke (1985), o que gera utilidade para o consumidor representativo não é o gasto total em bens duráveis e não-duráveis, mas sim o gasto com bens nãoduráveis e o estoque de bens duráveis, respectivamente.

As comparações dos gastos de duráveis e não-duráveis indicam que variações do primeiro têm maior amplitude do que às do segundo, implicando num comportamento cíclico mais pronunciado para o gasto de duráveis, o que poderia ser um elemento importante no entendimento do ciclo de negócios brasileiro, objeto de estudo de uma literatura crescente no Brasil. Dados os resultados prévios da literatura brasileira de consumo, quando se investigou a existência de mercados incompletos de crédito, parte desse comportamento cíclico pode estar associado à restrições à liquidez no financiamento de bens duráveis, assim como se encontrou previamente para o consumo total.

Nesse artigo, investigamos amplamente as propriedades dos gastos com bens duráveis no Brasil, tendo como ponto de partida um modelo integrado para consumo de bens duráveis, não-duráveis, e renda. A aplicação do método generalizado dos momentos (GMM), às equações de Euler do problema do consumidor representativo, possibilita testar diretamente a separabilidade da função utilidade entre estes dois tipos de gasto, questão fundamental para a correta avaliação da literatura corrente sobre o consumo no Brasil. Nossos testes de separabilidade são 
inspirados em Bernanke (1985), que considera a utilidade não-separável no estoque de bens duráveis e no consumo de não-duráveis, embora a função de utilidade utilizada aqui seja mais geral do que a forma quadrática usada naquele estudo.

A partir dos resultados encontrados nos testes de separabilidade, que não a rejeitaram, investigamos as propriedades estocásticas das séries brasileiras de consumo de duráveis e não-duráveis. Estas são estudadas a partir de modelos de otimização intertemporal que consideram o bem-estar associado aos serviços advindos do estoque de bens de consumo duráveis. Tais modelos mostram que o comportamento do consumo de bens duráveis é diferente do consumo de bens não-duráveis, encontrando diferentes restrições testáveis para cada tipo de gasto. Ao que sabemos, trata-se de um estudo pioneiro e necessário sobre o assunto no Brasil. Nele investigamos duas questões centrais à essa literatura: a comparação da dinâmica de curto e longo prazos entre os gastos de não-duráveis, duráveis e renda (Mankiw, 1981, Bernanke, 1985, Gali, 1993, Vahid e Engle, 1997) e possível existência de restrições à liquidez (Campbell, 1987, Bacchetta e Gerlach, 1997) no consumo de duráveis.

Além do importante resultado de encontrarmos separabilidade entre duráveis e não-duráveis, que validam estudos sobre consumo anteriores ao nosso, concluímos que há uma relação de longo prazo (cointegração) entre o consumo de duráveis, não-duráveis, e renda. No curto prazo, essas três séries exibem também uma dinâmica comum (têm um ciclo comum) que pode ser explicada em parte pela existência de restrição à liquidez no Brasil. Essa última evidência sugere que políticas que visam reduzir a restrição de crédito podem ter um impacto grande sobre o consumo, e, por conseqüência, sobre o bem-estar dos consumidores. Além disso, ao comparar nossos resultados atuais de restrição à liquidez aos resultados prévios de Reis et alii (1998), Issler e Rocha (2000) e Gomes (2004) encontramos evidência, ainda maior, a favor da hipótese de restrição ao crédito.

O resto desse estudo está dividido da seguinte forma: a próxima seção apresenta a teoria e as possíveis proposições testáveis, incluindo resultados empíricos obtidos com dados brasileiros e norte-americanos. A terceira seção apresenta a base de dados e a quarta os resultados empíricos. Por fim, a quinta seção apresenta as conclusões. 


\section{Teoria Econômica e Restrições Testáveis}

\subsection{Decisão de consumo}

Hall (1978) considera o problema de otimização no qual o consumidor escolhe o seu consumo de bens não-duráveis, $c n_{t}$, sujeito a uma restrição orçamentária. A equação de Euler é:

$$
u^{\prime}\left(c n_{t}\right)=E_{t}
$$

em que $\beta$ é a taxa de desconto intertemporal da utilidade futura, e $r_{t+1}$ é a taxa de retorno real da riqueza. Sob a hipótese de que $\beta\left(1+r_{t+1}\right)=1, \forall t$ :

$$
u^{\prime}\left(c n_{t+1}\right)=u^{\prime}\left(c n_{t}\right)+\epsilon_{t+1},
$$

em que $E_{t}\left(\epsilon_{t+1}\right)=0$, i.e., $\epsilon_{t+1}$ é uma inovação. Nesse caso, $u^{\prime}\left(c n_{t+1}\right)$ é uma martingala. Se supusermos, adicionalmente, que a utilidade é quadrá tica em $c n_{t}$, então a propriedade de martingala se estende à $c n_{t+1}$, de modo que $E_{t}\left(\Delta c n_{t+1}\right)=$ 0. i.e., $\Delta c n_{t+1}$ é imprevisível, ${ }^{1}$ o que é uma hipótese testável a partir do uso de técnicas econométricas.

Mankiw (1981) é o primeiro a modelar a decisão de gastos em bens duráveis em um arcabouço semelhante ao de Hall, no qual o agente decide o gasto em bens duráveis de forma a maximizar sua utilidade intertemporal, ${ }^{2}$ sujeito, além da restrição orçamentária, a uma lei de movimento do estoque de bens duráveis, $k_{t+1}=(1-\delta) k_{t}+c d_{t+1}$, em que $c d_{t+1}$ é o gasto em bens duráveis. Considerando as hipóteses de separabilidade da utilidade em $k_{t}$ e $c n_{t}$, de utilidade quadrática em $k_{t}$, e de $\beta\left(1+r_{t+1}\right)=1 \forall t$, é possível mostrar que

$$
\begin{aligned}
k_{t+1} & =k_{t}+\epsilon_{t+1} \mathrm{e} \\
c d_{t+1} & =c d_{t}+\epsilon_{t+1}-(1-\delta) \epsilon_{t},
\end{aligned}
$$

\footnotetext{
${ }^{1}$ Flavin (1981) demonstra que esse resultado pode ser obtido ao se considerar explicitamente a Teoria da Renda Permanente e a hipótese de expectativas racionais. A renda permanente, $y_{t}^{P}$, é dada por: $y_{t}^{P}=r\left[A_{t}+\sum_{s=0}^{\infty}\left(\frac{1}{1+r}\right)^{s+1} E_{t}\left(y_{t+s}\right)\right]$ em que $A_{t}$ e $y_{t}$ são, respectivamente, a riqueza e a renda do trabalho no período $t$.

${ }^{2}$ Em Mankiw (1981) a utilidade é função do serviço proporcionado pelo montante de estoque de bens duráveis que o agente possui. Esse autor considera ainda que o serviço é proporcional ao próprio estoque e portanto a função de utilidade pode ser escrita como função do estoque de bens duráveis.
} 
em que $E_{t}\left(\epsilon_{t+1}\right)=0$, i.e., $\epsilon_{t+1}$ é uma inovação. Neste caso, a variação do estoque de bens de consumo duráveis é uma inovação e a variação do gasto de duráveis é um processo $M A(1)$, que se constituem em restrições testáveis a partir do uso de técnicas econométricas.

Nos modelos de Hall (1978) e Mankiw (1981) os gastos de não-duráveis e de duráveis são modelados separadamente de modo que, necessariamente, uma hipótese de separabilidade na função de utilidade é adotada. Bernanke (1985) é o primeiro a modelar conjuntamente a decisão de gastos em bens duráveis e nãoduráveis. Esta forma de tratar o problema torna-se mais relevante quanto maior for a interdependência entre a decisão de gasto em bens duráveis e não-duráveis. Seguindo a tradição de Hall (1978) e Mankiw (1981), Bernanke (1985) considera a seguinte função utilidade, quadrática no consumo de duráveis e no estoque de não-duráveis,

$u\left(c n_{t}, k_{t}, k_{t+1}\right)=-\frac{1}{2}\left(\overline{c n}-c n_{t}\right)^{2}-\frac{a}{2}\left(\bar{k}-k_{t}\right)^{2}-m\left(\overline{c n}-c n_{t}\right)\left(\bar{k}-k_{t}\right)-\frac{d}{2}\left(k_{t+1}-k_{t}\right)^{2}$

em que o último termo do lado direito modela um custo de ajustamento no estoque de bens de consumo duráveis. A não separabilidade no gasto em bens de consumo não-duráveis e no estoque de bens duráveis se deve ao parâmetro $m$. Quando há separabilidade $m=0$, o que pode ser testado econometricamente. As equações de Euler desse problema são,

$$
\begin{gathered}
\overline{c n}-c n_{t}+m\left(\bar{k}-k_{t}\right)=\beta E_{t}\left\{\left(1+r_{t}\right)\left[\overline{c n}-c n_{t+1}+m\left(\bar{k}-k_{t+1}\right)\right]\right\} \\
-d\left(k_{t+1}-k_{t}\right)=\beta E_{t}\left\{\left[\bar{c}-c n_{t+1}+m\left(\bar{k}-k_{t+1}\right)\right]\right. \\
\left.\left[\left(1+r_{t}\right) p_{t}^{k}-(1-\delta) p_{t+1}^{k}\right]\right\}-\beta E_{t}\left[a\left(\bar{k}-k_{t+1}\right)+\right. \\
\left.m\left(\bar{c}-c n_{t+1}\right)+d\left(k_{t+2}-k_{t+1}\right)\right]
\end{gathered}
$$

em que $p_{t+1}^{k}$ é o preço relativo de duráveis em unidade de bens não-duráveis.

Uma implicação desse modelo, fruto da análise da equação (2) é que, se $\beta(1+r)=1$,

$$
\left(c n_{t+1}-m k_{t+1}\right)=\left(c n_{t}+m k_{t}\right)+\epsilon_{t+1}
$$

em que $E_{t}\left(\epsilon_{t+1}\right)=0$, i.e., $\epsilon_{t+1}$ é uma inovação. Nesse caso, existe uma combinação linear do gasto de não-duráveis e do estoque de duráveis que é uma martingala. 
Isto implica a existência de um ciclo comum para $\Delta c n_{t+1}$ e $\Delta k_{t+1}$, com coeficiente $(1,-m)$; ver Vahid e Engle (1997). Note que, quando há separabilidade $m=0$ e voltamos ao caso de Hall (1978) no qual a primeira diferença do gasto de nãoduráveis é imprevisível.

Considerando uma função de utilidade mais geral do que a usada por Bernanke, como, por exemplo, a do tipo elasticidade de substituição constante (CES), $u\left(c n_{t}, k_{t}\right)=\left(c n_{t}^{\rho}+\alpha k_{t}^{\rho}\right)^{\frac{1}{\rho}}$, as equações de Euler em (2) e (3) tornam-se,

$$
\begin{gathered}
E_{t}\left[\beta\left(c n_{t+1}^{\rho}+\alpha k_{t+1}^{\rho}\right)^{\frac{1-\rho}{\rho}} c n_{t+1}^{\rho-1}\left(1+r_{t}\right)\right]=\left(c n_{t}^{\rho}+\alpha k_{t}^{\rho}\right)^{\frac{1-\rho}{\rho}} c n_{t}^{\rho-1} \\
E_{t}\left\{\begin{array}{c}
\left(c n_{t+1}^{\rho}+\alpha k_{t+1}^{\rho}\right)^{\frac{1-\rho}{\rho}}\left[c n_{t+1}^{\rho-1} p_{t}^{k}\left(1+r_{t}\right)-\alpha k_{t+1}^{\rho-1}\right]+ \\
-\beta\left(c n_{t+2}^{\rho}+\alpha k_{t+2}^{\rho}\right)^{\frac{1-\rho}{\rho}} c n_{t+2}^{\rho-1} p_{t+1}^{k}(1-\delta)\left(1+r_{t+1}\right)
\end{array}\right\}=0
\end{gathered}
$$

Nesse caso, a hipótese de separabilidade entre a decisão de gasto em bens duráveis e não-duráveis pode ser examinada facilmente testando-se a hipótese nula de que $\rho=1$.

Mais recentemente, autores como Gali (1993) e Vahid e Engle (1997) modelam conjuntamente a decisão de consumo de bens duráveis, não-duráveis, impondo a separabilidade a priori. Para tanto, assumem uma função utilidade quadrática que não inclui um termo cruzado entre $c n_{t}$ e $k_{t}$. Vahid e Engle (1997) consideram a seguinte função de utilidade:

$$
u_{t}=u\left(c n_{t}, k_{t}\right)=\alpha_{1} c n_{t}-\frac{1}{2} b_{1} c n_{t}^{2}+\alpha_{2}\left(\tau k_{t}\right)-\frac{1}{2} b_{2}\left(\tau k_{t}\right)^{2},
$$

em que $\tau k_{t}$ representa o fluxo de serviços advindo do estoque de bens duráveis em t. Assumindo que $\beta(1+r)=1$ a solução do problema de otimização é descrita por:

$$
\begin{aligned}
c n_{t} & =\frac{\alpha_{1}}{b_{1}}-\frac{1}{b_{1}} \varkappa_{t}, \\
k_{t} & =\frac{\alpha_{2}}{\tau b_{2}}-\frac{\pi}{\tau^{2} b_{2}} \lambda_{t}+\frac{\pi \beta(1-\delta)}{\tau^{2} b_{2}} E_{t}\left(\varkappa_{t+1}\right), \\
\varkappa_{t} & =\varkappa_{t-1}+\varepsilon_{t},
\end{aligned}
$$

em que $\varepsilon_{t}$ é uma inovação, $\varkappa_{t}$ é o valor da utilidade marginal da riqueza em $t$, e $\pi$ é o preço relativo de duráveis em termos de não-duráveis. Usando a lei de movimento para o estoque de bens de consumo duráveis, e a equação de Euler (7), temos: 


$$
c d_{t}=\frac{\delta \alpha_{2}}{\tau b_{2}}+\rho \varkappa_{t}-(1-\delta) \rho \varkappa_{t-1}
$$

em que $\rho=-\frac{\pi[1-\beta(1-\delta)]}{\tau^{2} b_{2}}$.

Há vários pontos a notar. Primeiro, tanto $c n_{t}$ quanto $c d_{t}$ contêm $\varkappa_{t}$, que é um processo com raiz unitária (integrado de ordem um). Logo, ambos têm raiz unitária e cointegram. Pode-se mostrar que o vetor de cointegração é $\frac{1}{1-\delta}\left(b_{1} \delta, \rho^{-1}\right)$; ver Gali (1993). Segundo, o consumo de bens não-duráveis é uma martingala, como em Hall (1978) e Flavin (1981): $E_{t}\left(c n_{t+1}\right)=c n_{t}$ e $\Delta c n_{t}=$ $-\frac{1}{b_{1}} \varepsilon_{t}$. Terceiro, a primeira diferença do consumo de duráveis é um $M A(1)$, como em Mankiw (1981): $\Delta c d_{t}=\rho \varepsilon_{t}-(1-\delta) \rho \varepsilon_{t-1}$. O fato de que $\Delta c n_{t}$ é uma inovação, e de que $\Delta c d_{t}$ é uma $M A(1)$, implica que qualquer combinação linear, não nula, entre a primeira diferença do gasto em bens duráveis e não-duráveis resulta em um processo $M A(1)$, isto é, para $\gamma \neq 0, \Delta c n_{t}-\gamma \Delta c d_{t} \sim M A(1){ }^{3}$ Por fim, é possível mostrar que se for imposta a condição de que a sequência da riqueza real é limitada, ${ }^{4}$ o consumo de duráveis, não-duráveis e renda cointegram; ver Vahid e Engle (1997).

\subsection{Restrição à liquidez}

Campbell (1987) propuseram uma equação de teste para restrição à liquidez quando se supõe uma economia na qual existem dois tipos de consumidores: um consome a totalidade de sua renda corrente, de acordo com uma equação de consumo do tipo Keynesiana (sendo restrito à liquidez), e outro consome a sua renda permanente, de acordo com Hall (1978) e/ou Flavin (1981), ou mesmo levando-se em conta modelos mais elaborados para o crescimento do consumo, baseados na log-linearização da equação de Euler, na qual a taxa de crescimento do consumo depende do retorno real da riqueza, além de sua incerteza.

Considerando o modelo de Hall (1978) como benchmark de otimalidade, a existência de otimalidade (ou a inexistência de restrição à liquidez) pode ser testada através da hipótese nula de que $\lambda=0$ na equação de teste:

$$
\Delta c n_{t}=\lambda \Delta y_{t}+\varepsilon_{t}
$$

\footnotetext{
${ }^{3} \mathrm{Na}$ prática, caso as séries de consumo de bens duráveis e não-duráveis possuam componente cíclico e ainda assim esta relação persistir, dizemos que há um ciclo codependente entre estas séries. No apêndice apresentamos uma breve revisão do conceito de ciclos codependentes.

${ }^{4}$ Com isso, vale trivialmente uma condição de transversalidade, o que gera uma restrição de longo prazo entre a renda e o consumo.
} 
obtida quando se considera a heterogeneidade dos dois tipos de agentes na economia. Caso só existam agentes otimizadores, $\lambda=0$, sendo que $\lambda$ é a proporção da renda total pertencente aos consumidores com restrição à liquidez. Caso contrário, $\lambda \neq 0$, podemos não só verificar a inexistência de optimalidade, como também estimar a sua importância através do termo $\lambda$.

Quando consideramos o modelo de Mankiw (1981) como benchmark de otimização, a primeira diferença do consumo de bens duráveis é um $M A(1), \Delta c d_{t}=$ $-(1-\delta) \varepsilon_{t-1}+\varepsilon_{t}$, e a equação de teste é:

$$
\Delta c d_{t}=\lambda \Delta y_{t}+(1-\lambda)\left[(1-\delta) \varepsilon_{t-1}+\varepsilon_{t}\right]
$$

em que $\lambda$ é a proporção da renda que pertence a consumidores que não tem acesso ao crédito para consumir bens duráveis. Assim, sob a hipótese nula, $\lambda=0$ e os indivíduos têm pleno acesso ao crédito. Vale notar que esta equação consiste em um teste de ciclos codependentes entre $\Delta c d_{t}$ e $\Delta y_{t}$, i.e., a existência de uma combinação linear destas séries que é um processo $M A(\cdot)$ finito. Quando consideramos, adicionalmente, a possibilidade da equação de Euler envolver o retorno dos ativos, como em Campbell (1987), temos:

$$
\Delta c d_{t}=\lambda \Delta y_{t}+(1-\lambda) \theta r_{t}+(1-\lambda)\left[(1-\delta) \varepsilon_{t-1}+\varepsilon_{t}\right]
$$

que envolve ciclos codependentes entre $\Delta c d_{t}, \Delta y_{t}$ e $r_{t}$.

Em todas as equações de teste (10), (11) e (12), há a necessidade de se usar técnicas de variáveis instrumentais para estimar os parâmetros de interesse, pois a renda corrente é, em geral, correlacionada com a surpresa no gasto dos consumidores que não estão sujeitos à restrição de liquidez. Devido ao componente $M A(1)$ na equação (11), a escolha de instrumentos tem que ser defasada de pelo menos dois períodos.

Bacchetta e Gerlach (1997) modificam levemente a equação de teste proposta por Campbell (1987), usando:

$$
\Delta c n_{t}=\alpha+\theta E_{t-1}\left(\Delta X_{t}\right)+\varepsilon_{t}
$$

em que $X_{t}$ é composto pelo logarítmo da renda real disponível, variáveis de crédito imobiliário, variáveis de crédito do consumidor e diferença entre taxa de captação e empréstimo do mercado financeiro (ao invés da própria taxa de juros). Mais uma vez, há a necessidade de se usar técnicas de variáveis instrumentais para se estimar a equação de teste (13). 


\subsection{Evidências empíricas prévias para o Brasil}

Cavalcanti (1993) testa implicações da Teoria da Renda Permanente e a possibilidade de existência de restrição à liquidez, utilizando dados trimestrais de consumo agregado e PIB, per capita brasileiro, de 1980 a 1989. Esse autor encontra evidências de que estas séries são não estacionarias, porém não avalia se há cointegração entre elas. Com respeito ao teste de restrição à liquidez, utilizando estimadores de variáveis instrumentais, ele encontra evidência de que $32 \%$ da renda pertence a consumidores restritos.

Reis et alii (1998) estudam várias séries brasileiras anuais de consumo e renda agregados, per capita, datadas de 1947 a 1994, bem como séries trimestrais de janeiro de 1975 a abril de 1994. Não rejeitam a hipótese nula de raiz unitária para essas séries. Através da análise de cointegração, investigam a existência de relações de longo prazo entre consumo e renda, encontrando evidências a favor de um vetor de cointegração. Na maioria dos casos, não rejeitam a hipótese nula deste vetor ser $(1,-1)^{\prime}$ como previsto pela Teoria da Renda Permanente. Em seguida, utilizando metodologia proposta por Campbell (1987), os autores estimam que aproximadamente $80 \%$ da renda no Brasil pertence a consumidores que estão restritos a consumir apenas a sua renda corrente, número bastante superior ao encontrado por Cavalcanti (1993). Essa diferença é creditada ao conjunto de instrumentos usados na estimativa de (10), sendo que, por problemas de agregação temporal, a escolha de Reis et alii (1998) deve ser privilegiada. 5 Posteriormente, Issler e Rocha (2000) ratificam os resultados de Reis et alii (1998) ao estimarem uma proporção de $74 \%$, encontrando também evidências de que consumo e renda cointegram. ${ }^{6}$

Uma estimativa de $\lambda$ significativamente diferente de zero nas estimativas a partir de variáveis instrumentais e a validade dos testes de sobreidentificação implicam que o consumo e a renda agregados possuem um ciclo comum. Logo, o consumo agregado possui um componente cíclico. A despeito desses autores usarem o consumo agregado, eles consideram apenas o resultado de Hall (1978), segundo o qual o consumo não possui um componente cíclico e por isso rejeitam a Teoria da Renda Permanente. De fato, ao proceder a decomposição de Beveridge e Nelson (1981), Gomes (2004) estima que o consumo agregado anual de 1947 a 1999 para o Brasil possui um componente cíclico. Em uma tentativa de compatibilizar esta evidência empírica com o problema de otimização do consumidor, Gomes (2004) inclui a hipótese de formação de hábito. Com isso, foi derivada uma equação de

\footnotetext{
${ }^{5}$ Ver a discussão em Campbell (1987) e em Deaton (1992).

${ }^{6}$ Reis et alii (1998) encontram evidência de que o consumo é pouco sensível ao juros, mas tal investigação não foi conduzida por Issler e Rocha (2000).
} 
teste mais ampla que a proposta por Campbell (1987). Contudo ao proceder a sua estimativa é obtido um valor para $\lambda$ próximo de 0.85 e não se obtem evidência a favor da hipótese de formação de hábito. Com isso, Gomes (2004) conclui que a existência de um componente cíclico na série de consumo agregada não pode ser compatibilizada com o problema de otimização intertemporal do consumidor, em equilíbrio parcial, através da hipótese de formação de hábito. Ademais, uma grande parcela da renda pertence a consumidores que não têm acesso ao crédito. Não obstante essa evidência, a hipótese de otimização por parte do consumidor representativo ainda poderia ser mantida num arcabouço de equilíbrio geral, como notado por Issler e Vahid (2001).

Uma possível crítica que pode ser feita a todos os estudos prévios sobre o consumo no Brasil é o fato de que estes utilizam séries de consumo agregado, i.e., que somam o consumo de bens duráveis ao de não-duráveis. Como nota Bernanke (1985), o que gera utilidade para o consumidor representativo não é o gasto total em bens duráveis e não-duráveis, mas sim o gasto com bens não-duráveis e o estoque de bens duráveis, respectivamente. Ademais, do ponto de vista teórico, estas séries possuem comportamento diferenciado em modelos de equilíbrio parcial: a série de duráveis possui um componente cíclico, enquanto a de não-duráveis não o possui. Dessa forma, a soma das duas tem necessariamente um componente cíclico, o que não foi levado em conta nos estudos anteriores. Nesse sentido, as estimativas significativas de $\lambda$ podem ter se dado pela existência do componente cíclico da série de consumo de não-duráveis e não pela existência de restrição à liquidez. ${ }^{7}$ Os estudos anteriores podem ser validados se for rejeitada a hipótese de que a diferença do consumo de bens duráveis é um processo $M A(1)$, pois neste caso o consumo agregado não possui qualquer componente cíclico.

\footnotetext{
${ }^{7}$ Esta crítica não é pertinente no caso de Gomes (2004), uma vez que este autor considera um termo MA(1) na equação de teste.
} 


\section{Base de Dados e Análise Preliminar}

Para testar as implicações do modelo teórico são obtidos dados anuais para consumo de não-duráveis e de duráveis de 1970 a 1998 construidos por Ellery Jr. et alii (2002), ${ }^{8}$ em termos per capita. ${ }^{9}$ Como medida de renda, utilizamos a renda disponível bruta real, deflacionada pelo Deflator Implícito do PIB, em termos per capita, ambos disponibilizados pelo IPEADATA. ${ }^{10}$ Para construir a série de preço relativos de duráveis, usamos o índice IPA-DI para bens de consumo duráveis e de não-duráveis e por definição $p_{t}^{k}=\frac{I P A_{t}^{\text {duráveis }}}{I P A_{t}^{\text {duráveis }}}$, disponibilizado pelo FGVDADOS. Utilizamos ainda a série de juros real do CDB acumulado no ano, extraído da MACRODADOS.

A série de estoque de bens duráveis é gerada conforme metodologia apresentada no Apêndice A.3., segundo uma função de acumulação dos fluxos de consumo de bens duráveis. Nesta, é necessário fazer uma hipótese sobre o valor da taxa de depreciação acrescida da taxa de crescimento do consumo de bens duráveis, $\delta+g$. Os dados mostram que $g=0.033$ no período de análise. Consideramos cinco valores distintos para $\delta+g$, implicando nos seguintes valores para a taxa de depreciação $1.67 \%, 3.67 \%, 6.67 \%, 8.67 \%$ e $11.67 \% .11$

Abaixo na figura 1 apresentamos os gráficos das séries de consumo duráveis e não-duráveis, renda disponível em termos per capita, além do preço relativo de consumo de duráveis e não-duráveis e a taxa de juros.

\footnotetext{
${ }^{8}$ Ver apêndice A.2

${ }^{9}$ Os dados sobre população são provenientes do Instituto Brasileriro de Geografia e Estatística e se referem a população residente.

${ }^{10}$ Ver www.ipeadata.gov.br.

${ }^{11}$ Estudando o comportamento agregado do consumo no Brasil, através de uma economia artificial, Bugarin e Ellery Jr. (2002) utilizam uma taxa de depreciação para o estoque de capital igual a $10 \%$. No caso americano é comum utilizar uma taxa de depreciação de $3 \%$. Apesar de estarmos interessados na taxa de depreciação do estoque de bens duráveis, consideramos valores em torno de $10 \%$, privilegiando taxas menores, pois não esperamos que a taxa de depreciação no Brasil seja muito superior a taxa da economia americana.
} 
Figura 1
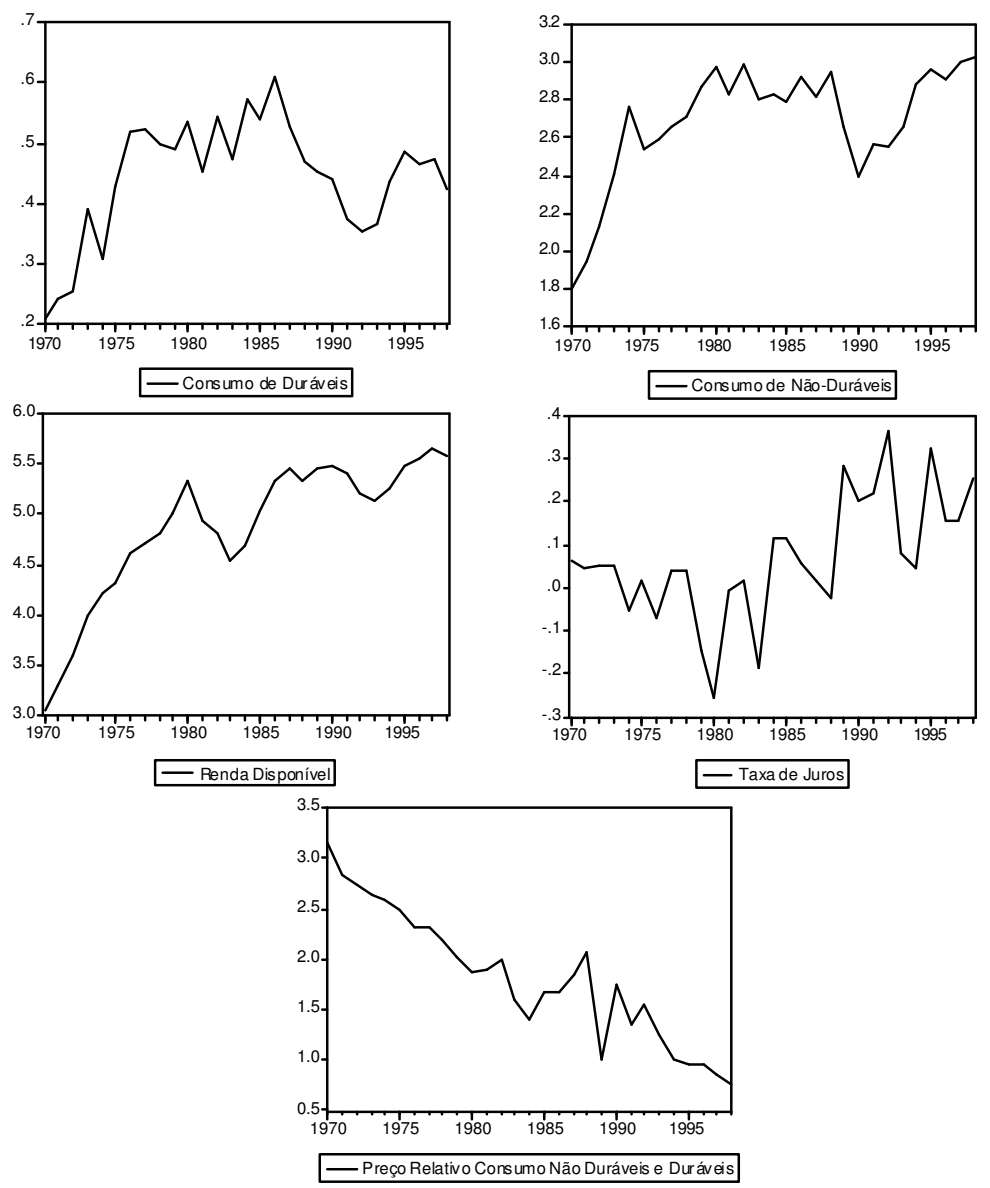
Por fim, apresentamos os resultados do teste ADF de raiz unitária das séries de taxa de juros e preço relativo. Como podemos observar na tabela 1 os resultados sugerem que estas séries são estacionárias, no nível de significância de 5\%. Como avaliamos se a renda, o consumo de bens duráveis e não-duráveis cointegram, para estas séries o teste de raiz unitária univariado é dispensável. Isto porque o teste de cointegração pode ser visto também como um teste multivariado de raiz unitária, pois se estas séries são estacionárias devemos estimar 3 vetores de cointegração. De fato, se existe uma relação desta natureza entre estas séries utilizar um teste de raiz unitária univariado consiste em um erro de especificação.

Tabela 1

Testes de raiz unitária

\begin{tabular}{lccc}
\hline \multicolumn{1}{c}{ Série } & Estatística ADF & p-valor & $\mathrm{n}^{\circ}$ de defasagens \\
\hline Taxa de juros & -4.14 & 0.0151 & 0 \\
Preço relativo & -5.15 & 0.0014 & 0 \\
\hline Os testes incluíram constante e tendência. \\
O número de defasagens incluídas no teste \\
foi escolhido usando o critério de informação de Akaike.
\end{tabular}

\section{Estimações e Resultados}

A primeira questão a ser discutida é a interdependência entre a decisão de gasto em bens duráveis e não-duráveis: a separabilidade entre o gasto de não-duráveis e o estoque de duráveis na função utilidade do consumidor representativo. Para examinar tal questão, estimamos por GMM as equações (4) e (5) e testamos a hipótese nula que $\rho=1$. Como dito anteriormente, consideramos cinco valores distintos para a taxa de depreciação, porém como os resultados são muito semelhantes reportamos apenas o caso no qual $\delta=0.067$. Utilizamos 8 conjuntos distintos de instrumentos formados por variáveis que pertencem ao conjunto de informação do consumidor. Usamos o teste de sobreidentificação de Hansen (estatística $T \times J$ ) para testar a especificação do modelo utilizado. A tabela 2 mostra os coeficientes estimados para o fator de desconto intertemporal, $\beta$, o parâmetro do estoque de bens duráveis da função de utilidade, $\alpha$ e a taxa de substituição intertemporal da função CES, $\rho$. Procedemos então o teste de Wald para avaliar a hipótese nula $\rho=1$. 
Tabela 2

Estimação por GMM das equações (4) e (5) para $\delta=0.067$

\begin{tabular}{|c|c|c|c|c|c|c|c|c|}
\hline \multirow[t]{2}{*}{ Modelo } & \multirow[t]{2}{*}{ Instrumentos } & \multicolumn{3}{|c|}{$\begin{array}{l}\text { Coeficientes } \\
\text { Estimados }\end{array}$} & \multicolumn{2}{|c|}{$\begin{array}{c}\text { Teste de } \\
\text { Separabilidade } \\
(\mathrm{H} 0: \mathrm{r}=1)\end{array}$} & \multicolumn{2}{|c|}{$\begin{array}{c}\text { Teste de } \\
\text { Sobreidentificação }\end{array}$} \\
\hline & & $\bar{\beta}$ & $\alpha$ & $\rho$ & $\begin{array}{c}\text { Estatística } \\
\text { teste }\end{array}$ & p-valor & $\begin{array}{c}\text { Estatística } \\
\text { TJ de Hansen }\end{array}$ & p-valor \\
\hline 1 & $\begin{array}{l}c d_{t}, c d_{t-1} \\
r_{t-1}, r_{t-2}\end{array}$ & $\begin{array}{l}0,9847^{* *} \\
(0.03068)\end{array}$ & $\begin{array}{c}0,1713 \\
(0.10495)\end{array}$ & $\begin{array}{c}1,9261^{*} \\
(0.76001)\end{array}$ & 1,4847 & 0,2230 & 6,7174 & 0,4589 \\
\hline 2 & $c d_{t-1}, y_{t-1}, y_{t-2}$ & $\begin{array}{l}0,9972^{* *} \\
(0.01859)\end{array}$ & $\begin{array}{c}0,1548 \\
(0.10310)\end{array}$ & $\begin{array}{c}1,3665 \\
(0.86991)\end{array}$ & 0,1775 & 0,6736 & 4,6851 & 0,4555 \\
\hline 3 & $y_{t-1}$ & $\begin{array}{l}0,9924^{* *} \\
(0.01905)\end{array}$ & $\begin{array}{c}0,2221 \\
(0.12240)\end{array}$ & $\begin{array}{c}1,1178 \\
(0.89502)\end{array}$ & 0,0173 & 0,8953 & 3,0488 & 0,0808 \\
\hline 4 & $\begin{array}{c}c n_{t-1}, y_{t}, y_{t-1} \\
p_{k t-1}, r_{t-1}\end{array}$ & $\begin{array}{l}0,9628^{* *} \\
(0.01530)\end{array}$ & $\begin{array}{l}0,3088^{* *} \\
(0.07794)\end{array}$ & $\begin{array}{l}1,6403^{* *} \\
(0.35852)\end{array}$ & 3,1898 & 0,0741 & 8,0226 & 0,5319 \\
\hline 5 & $c_{n t-1}, y_{t-1}, p_{k t-1}$ & $\begin{array}{l}0,9918^{* *} \\
(0.01750)\end{array}$ & $\begin{array}{l}0,2532^{* *} \\
(0.09275)\end{array}$ & $\begin{array}{l}1,3279^{* *} \\
(0.48153)\end{array}$ & 0,4637 & 0,4959 & 6,5895 & 0,6798 \\
\hline 6 & $k_{t-1}, y_{t-1}, y_{t-2}$ & $\begin{array}{l}0,8990^{* *} \\
(0.02316)\end{array}$ & $\begin{array}{l}0,2142^{* *} \\
(0.08339)\end{array}$ & $\begin{array}{l}1,5603^{*} \\
(0.58053)\end{array}$ & 0,9316 & 0,3344 & 5,6613 & 0,8950 \\
\hline 7 & $c d_{t}, c d_{t-1}, c d_{t-2}, y_{t}$ & $\begin{array}{l}0,9806^{* *} \\
(0.01366)\end{array}$ & $\begin{array}{c}0,0381 \\
(0.06181)\end{array}$ & $\begin{array}{c}2,1086 \\
(1.89014)\end{array}$ & 0,3440 & 0,5575 & 7,6738 & 0,1752 \\
\hline 8 & $k_{t-1}, k_{t-2}, r_{t-1}$ & $\begin{array}{l}0,9985^{* *} \\
(0.01935)\end{array}$ & $\begin{array}{c}0,2098^{*} \\
(0.08919)\end{array}$ & $\begin{array}{l}1,6139^{* *} \\
(0.49010)\end{array}$ & 1,5693 & 0,2103 & 5,7577 & 0,3305 \\
\hline & média & 0,9759 & 0,1965 & 1,5827 & & & & \\
\hline
\end{tabular}

*(**) denota rejeição de que o coeficiente é significativo ao nível de 5\%(1\%) de significância; desvios-padrões entre parênteses.

O teste de sobreidentificação de Hansen não rejeita a hipótese nula de que as condições de momento são válidas para todos os grupos de instrumentos utilizados. Os valores estimados para $\beta$ são próximos a 1 e significativamente diferentes de zero para todos os conjuntos de instrumentos, com mediana 0.9882 . Isto representa uma taxa de desconto intertemporal de aproximadamente $1.19 \%$ ao ano.

Para todos os grupos de instrumentos testados não é rejeitada a hipótese nula de que $\rho=1$, ou seja, não se rejeita a separabilidade entre o gasto de não-duráveis e o estoque de duráveis na função utilidade do consumidor representativo. Esse resultado tem grande importância na medida em que corrobora todo um esforço passado de pesquisa que considerava implicitamente esta hipótese de separabilidade, e que estariam irremediavelmente condenados caso a separabilidade não fosse verificada. 
Cabe ressaltar que, para o caso em que $\rho=1$, o coeficiente $\alpha$ da função CES equivale à taxa marginal de substituição entre consumo de não-duráveis e estoque de duráveis. A taxa marginal de substituição estimada para os grupos de instrumentos utilizados é estatisticamente inferior a unidade, com mediana de 0.212, dentre nossas estimativas. Logo, o consumidor brasileiro é indiferente entre 0.212 unidades de estoque de duráveis e uma unidade de bem de consumo nãodurável.

O resultado do teste de separabilidade nos permite seguir considerando as restrições testáveis propostas por Vahid e Engle (1997) e Gali (1993), apresentadas na seção 2. A importância de se avaliar tais restrições é o fato de expressarem restrições de curto e longo prazo sobre a dinâmica das séries de gasto de duráveis e não-duráveis.

A tabela 3 apresenta a estimativa do modelo $M A(1)$ de Mankiw (1981) para a primeira diferença do gasto com duráveis. Apesar do coeficiente ser negativo, como previsto pela teoria, o coeficiente da $M A(1)$ não foi significativamente diferente de zero aos níveis de significância de $5 \%$ ou $10 \%$.

Tabela 3

Estimativa do Modelo de Mankiw (1981) $\Delta c d_{t}=\varepsilon_{t}-(1-\delta) \varepsilon_{t-1}$

\begin{tabular}{lcc}
\hline & Variável Dependente: & D(Consumo de Duráveis) \\
\hline Regressor & $(1)$ & $(2)$ \\
\hline Constante & 0,0078 & - \\
& $(0.7558)$ & \\
$-(1-\delta)$ & $-0,2211$ & $-0,2005$ \\
& $(-1.0504)$ & $(-1.0958)$ \\
\hline
\end{tabular}

Nota: Estatística $t$ entre parênteses. Utilizamos o método proposto por Newey e West (1987) para estimar a matriz de covariância dos resíduos.

Os resultados da tabela 3 sugerem que o consumo de não-duráveis é um passeio aleatório. Nesse caso, se a teoria sobre o gasto de não-duráveis fosse válida, a soma do gasto de duráveis e não-duráveis seria um passeio aleatório. Logo, o fato do termo $M A(1)$ ser não significativo pode validar estudos anteriores que usam o passeio aleatório como benchmark para a série de consumo agregado, seguindo Hall (1978). Além disso, a rejeição da hipótese de que o consumo total não é um passeio aleatório, obtida por esses autores, não pode ser atribuída a um problema de viés de agregação. 
Para testar as restrições do modelo de Vahid e Engle (1997), de que, para $\gamma \neq 0, \Delta c n_{t}-\gamma \Delta c d_{t} \sim M A(1)$, e, de que, o gasto de duráveis e não-duráveis são cointegrados, estimamos um VAR contendo o consumo de bens duráveis e não-duráveis. Para escolher a ordem deste VAR utilizamos um teste de Razão de Verosimilhança, aliado ao uso de critérios de informação. A especificação de um $V A R(1)$ parece ser um modelo razoável. Com base neste modelo realizamos o teste de cointegração de Johansen. Os resultados são apresentados na tabela 4 abaixo.

Tabela 4

Teste de Cointegração de Johansen Modelo VAR(1) para $c d$ e $c n$

\begin{tabular}{|c|c|c|c|c|c|}
\hline \multicolumn{6}{|c|}{ Teste do Posto de Cointegracãa sem restrição } \\
\hline Ho: $\mathrm{N}^{\circ}$ de Eq. & Autovalor & Teste c & Traço & Teste do m & z autovalor \\
\hline de Cointegração & & $\begin{array}{c}\text { Estatística de } \\
\text { teste }\end{array}$ & $\begin{array}{c}\text { Valor Crítico } \\
5 \%\end{array}$ & $\begin{array}{c}\text { Estatística de } \\
\text { teste }\end{array}$ & $\begin{array}{c}\text { Valor Crítico } \\
5 \%\end{array}$ \\
\hline Nenhuma & 0,313342 & 11,5564 & 12,53 & 10,5257 & 11,44 \\
\hline No máximo 1 & 0,036141 & 1,0306 & 3,84 & 1,0307 & 3,84 \\
\hline
\end{tabular}

Tanto o teste baseado na estatística do traço quanto na estatística do autovalor máximo não rejeitam a hipótese nula de não existência de cointegração, no nível de significância de 5\%. Há cointegração ao nível de 10\%, mas esse resultado não é inequívoco. Incluímos então a renda real no VAR, testando novamente a existência de cointegração, conforme sugere a teoria no caso da riqueza real ser limitada.

Usando o gasto de duráveis, não-duráveis, e renda, a ordem do VAR é escolhida em 3, após consulta a diversos testes de diagnóstico e critérios de informação. Procedemos então o teste de cointegração de Johansen (1988), apresentado na tabela 5 . 
Tabela 5

Teste de Cointegração de Johansen (1988) Modelo VAR(3) para cd, cn e $y$

\begin{tabular}{|c|c|c|c|c|c|}
\hline \multicolumn{6}{|c|}{ Teste do Posto de Cointegração sem restrição } \\
\hline \multirow{2}{*}{$\begin{array}{l}\text { Ho: } N^{\circ} \text { de Eq. } \\
\text { de Cointegração }\end{array}$} & \multirow{2}{*}{ Autovalor } & \multicolumn{2}{|c|}{ Teste do Traço } & \multicolumn{2}{|c|}{ Teste do max autovalor } \\
\hline & & Estatística de & Valor Crítico & Estatística de & Valor Crítico \\
\hline Nenhuma & 0,508875 & 26,6917 & 24,31 & 18,4875 & 17,89 \\
\hline No máximo 1 & 0,248983 & $8.2043 *$ & 12,53 & $7.4445 *$ & 11,44 \\
\hline No máximo 2 & 0,028798 & 0.7597 & 3.84 & 0.7597 & 3.84 \\
\hline \multirow{3}{*}{\multicolumn{6}{|c|}{$\begin{array}{l}*(* *) \text { denota rejeição da hipótese no nível de } 5 \%(1 \%) \text { de significância } \\
\text { Teste do traço indica não cointegração no nível de } 5 \% \text { de significância } \\
\text { Teste do Máximo autovalor indica não cointegração no nível de } 5 \% \text { de significância }\end{array}$}} \\
\hline & & & & & \\
\hline & & & & & \\
\hline & \multicolumn{4}{|c|}{ Coeficientes da Equação de Cointegração Normalizados } & \\
\hline & $\mathrm{CN}$ & $\mathrm{CD}$ & \multicolumn{2}{|c|}{ RENDADISP } & \\
\hline & \multirow[b]{3}{*}{ Dosyion } & $-2,682945$ & \multicolumn{2}{|l|}{$-0,295274$} & \\
\hline & & $(0.40866)$ & $(0.03788)$ & & \\
\hline & & \multicolumn{3}{|c|}{ Desvio-padrão entre parênteses } & \\
\hline
\end{tabular}

Tanto o teste baseado na estatística do traço como o teste baseado na estatística do auto-valor máximo não rejeitam a hipótese nula de que existe 1 vetor de cointegração, no nível de significância de $5 \%$. Sendo a ordem das variáveis do VAR $\mathrm{cn}_{t}$, $c d_{t}$ e $y_{t}$, respectivamente, o vetor de cointegração estimado é $(1,-2.6829,-0.2953)$, o que confirma uma das proposições testáveis de Vahid e Engle (1997). Utilizando uma série de consumo agregada, e uma medida da renda, Issler e Rocha (2000) e Reis et alii (1998) obtêm um resultado análogo, não rejeitando a existência de um vetor de cointegração entre consumo e renda.

Uma vez confirmado o comovimento de longo prazo entre as séries de consumo e renda, passamos a analisar a possibilidade de existir comovimentos de curto prazo nas mesmas, i.e., a existência de ciclos comuns ou de ciclos codependentes entre elas. ${ }^{12}$ A importância de avaliarmos se as séries possuem alguma característica comum de curto prazo está no fato de que o estudo da dinâmica de curto prazo do conjunto pode ser resumido ao estudo da dinâmica de apenas uma delas. Assim, se este for o caso, o comportamento de curto prazo da renda é decisivo para o entendimento do comportamento das séries de consumo, o que pode indicar a presença de restrição à liquidez no consumo, conforme argumentam Campbell (1987).

Testamos a presença de características comuns de curto prazo utilizando os testes de correlação canônica descritos em Vahid e Engle (1997). Usamos 4 defasagens da primeira diferença das séries de consumo de duráveis e não-duráveis e

\footnotetext{
${ }^{12}$ Estes conceitos são brevemente discutidos no apêndice A.1.
} 
renda além da primeira defasagem do termo de correção de erros como instrumentos para calcular as correlações canônicas. Os resultados estão expostos na tabela 6 abaixo.

Tabela 6

\begin{tabular}{|c|c|c|c|c|c|}
\hline \multicolumn{6}{|c|}{ Teste de Ciclos Comuns } \\
\hline \multirow{2}{*}{\multicolumn{2}{|c|}{$\begin{array}{c}\text { Ho: } 1 \text { Vetor de } \\
\text { Co-Característico }\end{array}$}} & \multirow{2}{*}{\multicolumn{2}{|c|}{$\begin{array}{l}\text { Ho: } 2 \text { Vetores de } \\
\text { Co-Característico }\end{array}$}} & \multirow{2}{*}{\multicolumn{2}{|c|}{$\begin{array}{l}\text { Ho: } 3 \text { Vetores de } \\
\text { Co-Característico }\end{array}$}} \\
\hline & & & & & \\
\hline Estatística de & Valor Crítico & Estatística de & Valor Crítico & Estatística de & Valor Crítico \\
\hline Teste & $5 \%$ & Teste & $5 \%$ & Teste & $5 \%$ \\
\hline 9,42 & 19,68 & 28,62 & 36,42 & $64.17^{*}$ & 54,57 \\
\hline \multicolumn{6}{|c|}{$*(* *)$ denota rejeição de Ho a $5 \%(1 \%)$ de significância } \\
\hline \multicolumn{6}{|c|}{ Teste de Ciclos Codependentes } \\
\hline \multirow{2}{*}{\multicolumn{2}{|c|}{$\begin{array}{c}\text { Ho: } 1 \text { Vetor de } \\
\text { Ciclo Codependente }\end{array}$}} & \multirow{2}{*}{\multicolumn{2}{|c|}{$\begin{array}{c}\text { Ho: } 2 \text { Vetores de } \\
\text { Ciclo Codependente }\end{array}$}} & \multirow{2}{*}{\multicolumn{2}{|c|}{$\begin{array}{c}\text { Ho: } 3 \text { Vetores de } \\
\text { Ciclo Codependente }\end{array}$}} \\
\hline & & & & & \\
\hline Estatística de & Valor Crítico & Estatística de & Valor Crítico & Estatística de & Valor Crítico \\
\hline Teste & $5 \%$ & Teste & $5 \%$ & Teste & $5 \%$ \\
\hline 8,95 & 19,68 & 25,98 & 36,42 & 49,13 & 54,57 \\
\hline
\end{tabular}

Ao contrário dos resultados encontrados por Vahid e Engle para o caso americano, não encontramos evidência a favor da existência de ciclos codependentes, uma vez que os testes não rejeitam a existência de ciclos comuns entre o consumo de duráveis, não-duráveis e renda, ao nível de significância de 5\%. Os testes apontam a existência de dois vetores co-característicos, isto é, existem 2 combinações lineares não-nulas e linearmente independentes entre as primeiras diferenças das séries que geram uma série que é uma inovação.

Os testes de restrição a liquidez para o gasto de duráveis são apresentados abaixo na tabela 7. Dado que o componente $M A(1)$ foi não significativo em todos os casos, optou-se por reportar apenas a equação de teste quando se elimina o termo $M A(1)$. As estimativas são feitas utilizando o método de variáveis instrumentais, sendo utilizado como instrumentos variáveis defasadas pelo menos em 2 períodos. Na tabela 7 reportamos os resultados utilizando 5 conjuntos distintos de instrumentos. Em todos os casos não é rejeitado o teste de sobre-identificação da validade dos instrumentos, no nível de significância de 5\%. As estimativas do coeficiente referente a renda é significativamente diferente de zero, ao nível de significância de 5\%, em quase todos os casos. Quanto ao coeficiente da taxa de juros, em todos os casos, sua estimativa é não significativa, ao nível de significância de $5 \%$. 
Tabela 7

Teste de Restrição à liquidez $\Delta c d_{t}=\lambda \Delta y_{t}+(1-\lambda) \theta r_{t}+(1-\lambda) \varepsilon_{t}$

\begin{tabular}{cccccc}
\hline Modelo & Instrumentos & $\lambda^{(a)}$ & $\theta^{(a)}$ & $(1-\lambda) \theta^{(a)}$ & $\begin{array}{c}\text { Teste de } \\
\text { Sobreidentificação } \\
T J^{(b)}\end{array}$ \\
\hline 1 & $\Delta c d_{t-2}, \Delta Y_{t-2}, \Delta c n_{t-2}, r_{t-2}$ & 0,3661 & $-0,2498$ & $-0,1583$ & 2,4308 \\
& & $(0,0055)$ & $(0,2878)$ & $(0,2604)$ & $(0,2966)$ \\
2 & $\Delta c d_{t-2}, \Delta Y_{t-2}, \Delta c d_{t-3}$, & 0,0866 & $-0,0321$ & $-0,0293$ & 3,2877 \\
& $\Delta Y_{t-3}, r_{t-2}$ & $(0,4573)$ & $(0,8430)$ & $(0,8399)$ & $(0,3494)$ \\
& $\Delta c d_{t-2}, \Delta Y_{t-2}, \Delta c d_{t-3}$, & 0,2024 & $-0,1346$ & $-0,1074$ & 4,3860 \\
4 & $\Delta Y_{t-3}, \Delta c n_{t-2}, r_{t-2}$ & $(0,0071)$ & $(0,1773)$ & $(0,1644)$ & $(0,3563)$ \\
& $\Delta c d_{t-2}, \Delta Y_{t-2}, \Delta c d_{t-3}, \Delta Y_{t-3}$, & 0,1875 & $-0,1528$ & $-0,1241$ & 4,2271 \\
5 & $\Delta c n_{t-2}, \Delta c n_{t-3}, r_{t-2}$ & $(0,0172)$ & $(0,1271)$ & $(0,1094)$ & $(0,5172)$ \\
& $\Delta c d_{t-2}, \Delta Y_{t-2}, p k_{t-2}, r_{t-2}$ & 0,2288 & $-0,1120$ & $-0,0864$ & 2,7443 \\
& & $(0,0207)$ & $(0,2164)$ & $(0,2134)$ & $(0,2536)$ \\
\hline
\end{tabular}

Nota: (a) entre parênteses o p-valor para o teste de significância do respectivo coeficiente;

(b) entre parênteses o p-valor para a estatística do teste de Hansen; Nas estimativas por

GMM utilizou-se o núcleo de Bartlett com janela de Andrews, além da opção de prewhitening.

A estimativa mediana de $\lambda$ é aproximadamente 0.20 , e significativa para a maioria dos instrumentos utilizados. Para comparar este valor com o valor obtido por Reis et alii (1998) de 0.8, por Issler e Rocha (2000) de 0.74 e por Gomes (2004) de 0.85 é preciso levar em conta que ambos trabalharam com uma série de consumo que agrega bens duráveis e não-duráveis, $c_{t}=c d_{t}+c n_{t}$. Como a razão $c d_{t} / c_{t}$ é bastante estável e possui média aproximadamente igual a 0.14 , multiplicando $\Delta c_{t}$ por este valor obtemos uma aproximação bastante razoável para $\Delta c d_{t}$. Como sabemos, multiplicar a variável dependente por uma constante implica que os coeficientes estimados também ficam multiplicados por esta constante. Isto posto, multiplicamos $0.8,0.74$ e 0.85 por 0.14 obtendo então as estimativas $0.11,0.10$ e 0.12 , respectivamente, valores inferiores a nossa estimativa mediana. ${ }^{13}$ Logo, $^{2}$ apesar de obtermos um coeficiente menor para a renda, obtivemos resultados mais fortes de restrição à liquidez do que os obtidos anteriormente. Isso não nos surpreende, pois usamos a série de gasto de duráveis, que, na maioria dos casos, requer maior crédito para que o gasto seja efetuado.

Dado que o coeficiente da taxa de juros é não-significativo em todas as estimativas da tabela 7, consideramos reestimar as mesmas equações omitindo o juros real. Os resultados são apresentados abaixo na tabela 8 .

\footnotetext{
${ }^{13}$ Como $c d_{t} / c_{t} \approx 0.14$, é possivel mostrar que $\Delta c d_{t} \approx 0.14 \Delta c_{t}$. Reis et alii (1998), Issler e Vahid (2001) e Gomes (2004) consideram $\Delta c_{t}=\lambda \Delta y_{t}+(1-\lambda) \varepsilon_{t}$. Multiplicando por 0.14 obtemos, $\Delta c d_{t} \approx 0.14 \lambda \Delta y_{t}+0.14(1-\lambda) \varepsilon_{t}$, dai fica claro a necessidade de multiplicar os valores obtidos por esses autores por 0.14 .
} 
Tabela 8

Teste de Restrição à liquidez $\Delta c d_{t}=\lambda \Delta y_{t}+(1-\lambda) \varepsilon_{t}$

\begin{tabular}{|c|c|c|c|}
\hline Modelo & Instrumentos & $\lambda^{(a)}$ & $\begin{array}{c}\text { Teste de } \\
\text { Sobreidentificação }^{(b)}\end{array}$ \\
\hline 1 & $\begin{array}{c}\Delta c d_{t-2}, \Delta Y_{t-2} \\
\Delta c n_{t-2}, r_{t-2}\end{array}$ & 0,2464 & 3,9820 \\
\hline 2 & $\begin{array}{c}\Delta c d_{t-2}, \Delta Y_{t-2}, \Delta c d_{t-3} \\
\Delta Y_{t-3}, r_{t-2}\end{array}$ & $\begin{array}{c}(0,0430) \\
0,0536\end{array}$ & $\begin{array}{c}(0,2634) \\
4,0423\end{array}$ \\
\hline 3 & $\begin{array}{c}\Delta c d_{t-2}, \Delta Y_{t-2}, \Delta c d_{t-3} \\
\Delta Y_{t-3}, \Delta c n_{t-2}, r_{t-2}\end{array}$ & 0,1141 & 7,9056 \\
\hline 4 & $\begin{array}{c}\Delta c d_{t-2}, \Delta Y_{t-2}, \Delta c d_{t-3} \\
\Delta Y_{t-3}, \Delta c n_{t-2}, \Delta c n_{t-3}, r_{t-2}\end{array}$ & 0,1263 & $\begin{array}{c}(0,1010) \\
7,8784\end{array}$ \\
\hline 5 & $\Delta c d_{t-2}, \Delta Y_{t-2}, p k_{t-2}, r_{t-2}$ & $\begin{array}{c}(0,0690) \\
0,2070 \\
(0,0448)\end{array}$ & $\begin{array}{c}(0,2471) \\
2,9833 \\
(0,3942)\end{array}$ \\
\hline
\end{tabular}

Nota: (a) entre parênteses o p-valor para o teste de significância do coeficiente da renda; (b) entre parenteses o p-valor para a estatística de teste do teste de sobreidentificação; Nas regressões utilizou-se o método proposto por Newey e West (1987) para estimar a matriz de covariância dos resíduos.

Observamos na Tabela 8 que em todos os casos não se rejeita o teste de sobreidentificação, no nível de significância de $5 \%$, por ém nos casos 2 e 3 não se rejeita que $\lambda$ seja nulo. A estimativa mediana de $\lambda$ é aproximadamente igual a $0.1 \dot{4}$. Se considerarmos apenas os conjuntos de instrumentos 1, 4 e 5 este valor torna-se aproximadamente 0.20. Mais uma vez, apesar de obtermos um coeficiente menor para a renda, obtivemos resultados mais fortes de restrição à liquidez do que os obtidos anteriormente. ${ }^{14}$

Vale notar que a equação estimada na tabela 8 descreve um ciclo comum entre o gasto de duráveis e a renda, refletindo a possível existência de restrição à liquidez. De acordo com essa visão, o comportamento cíclico do gasto de duráveis no Brasil pode ser fruto de restrição à liquidez e não do ajuste ótimo do estoque de duráveis às flutuações da renda permanente. Esse tipo de restrição, se relaxada, resultaria em um estoque maior de bens duráveis, e, por conseqüência, em um aumento de bem-estar para os consumidores brasileiros.

\footnotetext{
${ }^{14}$ Estimamos ainda o modelo $\Delta c d_{t}=\beta r_{t}+\varepsilon_{t}$, por ém em todos os casos a estimativa de $\beta$ é não significativa, no nível de $5 \%$ de significância. Por isso não reportamos estas estimativas.
} 


\section{Conclusões}

Neste artigo investigamos a evolução da renda, consumo de bens duráveis e nãoduráveis no Brasil, com enfoque especial sobre a decisão de gasto em bens duráveis. Primeiramente, avaliamos se existe separabilidade na função utilidade entre o gasto de não-duráveis e o estoque de duráveis. Esta investigação é muito importante para validar os estudos anteriores, uma vez que todos assumem implicitamente esta hipótese. O teste de hipótese, realizado com base nas equações de Euler do problema do consumidor, utilizando a função de utilidade do tipo CES, estimada a partir de GMM, não rejeita a separabilidade.

A partir do resultado de separabilidade, foi possível obter ainda os seguintes resultados: i) os testes de cointegração não rejeitam a hipótese da existência de um vetor de cointegração entre consumo de duráveis, não-duráveis e renda; ii) os testes de ciclo comum não rejeitam a existência de um ciclo comum entre o consumo de duráveis, não-duráveis e renda; iii) os testes de restrição à liquidez sugerem que não se pode rejeitar que o consumo de bens duráveis sofre os efeitos da restrição de crédito, com restrição superior à encontrada para o consumo total, conforme os resultados de Cavalcanti (1993), Reis et alii (1998), Issler e Rocha (2000) e Gomes (2004).

Os resultados i) e ii) acima sugerem que, no Brasil, o consumo de duráveis, não-duráveis e renda apresentam comovimentos de curto e longo prazo. O resultado iii) evidencia que uma fração de consumidores consome a sua renda corrente e outra suaviza seu consumo, de acordo com a Teoria da Renda Permanente. Em ambos os casos, esperamos que o consumo (de bens duráveis e não-duráveis) cointegre com a renda, logo não é possível interpretar o resultado i) acima como uma evidência a favor de qualquer um dos casos. No entanto, a existência de ciclo comum entre as séries em questão é uma evidência a favor da hipótese de restrição ao crédito. Tal interpretação é confirmada com base nas estimativas obtidas de $\lambda$. Assim, conjecturamos que a estimativa de um ciclo comum ao invés de um ciclo codependente, entre o consumo de duráveis, não-duráveis e renda, pode ser devida a existência de uma elevada restrição de crédito.

Lembramos que, se duas séries apresentam um ciclo comum, elas possuem funções resposta à impulso colineares, para todo $t$. Isto significa que choques na renda impactam o consumo de duráveis e de não-duráveis de forma imediata e similar. Por outro lado, se estas séries tivessem um ciclo codependente de ordem $s$, a função resposta a impulso das mesmas tornariam-se colineares apenas após $s$ períodos. Nestes primeiros $s$ períodos, o consumo reagiria de forma diferente da renda, devido ao comportamento otimizador do consumidor que suavizaria seu con- 
sumo, caso tivesse acesso ao crédito. Logo, se existisse crédito, não esperaríamos que choques na renda se transmitissem automaticamente para o consumo, seja de duráveis ou não-duráveis. Neste sentido, fica claro como é possível conciliar a rejeição da hipótese de ciclo codependente e a não rejeição da hipótese de restrição de crédito.

Enfim, se o consumo corrente é muito dependente da renda corrente ao invés da renda permanente, podemos concluir que: i) políticas econômicas que afetam a renda têm impacto direto sobre grande parcela do consumo; ii) políticas econômicas que visam expandir o crédito têm um impacto potencial significativo em aumentar o bem-estar dos consumidores.

As análises implementadas neste artigo são baseadas nas séries de consumo de duráveis e não-duráveis construídas por Ellery Jr. et alii (2002). Esses autores obtem essas séries com base em uma metodologia que faz uso da estabilidade da participação do consumo de duráveis no consumo total. Alguém poderia argumentar que essa forma de desagregação entre duráveis e não-duráveis introduz um viés nos resultados a favor da hipótese de ciclo comum, contudo acreditamos que a causalidade é inversa: a existência de um ciclo comum é que gera a estabilidade da participação de duráveis. Apesar disto, como tópico de pesquisa futuro pretendemos obter novas medidas para estas séries com o objetivo de averiguar se os nossos resultados são robustos. Porém, ao nosso ver, dentre as disponíveis a metodologia aqui empregada parece ser a mais razoável.

A investigação com dados diferentes dos atuais se justifica por mais uma razão: em frequência anual, não há uma sobreposição entre o período de decisão dos agentes econômicos e a frequência das observações. Esta sobreposição parece indicar o uso de dados mensais (ou talvez mesmo trimestrais). Nesses casos, teríamos que recorrer a proxies para os dados de consumo (consumo aparente). De qualquer forma, isso merece investigação futura. Um outro ponto que também merece investigação futura é seguir a linha de Ogaki e Reinhart (1998) na qual combina-se técnicas de cointegração e de método dos momentos para estimar em duas etapas a elasticidade intertemporal de substituição e outros parâmetros estruturais. 


\section{Referências}

Bacchetta, P. \& Gerlach, S. (1997). Consumption and credit constraints: International evidence. Journal of Monetary Economics, 40(2):207-238.

Bernanke, B. (1985). Adjustment costs, durable goods and aggregate consumption. Journal of Monetary Economics, 15:41-68.

Beveridge, S. \& Nelson, C. R. (1981). A new approach to decomposition of economic Time Series into permanent and transitory components with particular attention to measurement of the business cycle. Journal of Monetary Economics, $7(2): 151-174$.

Bugarin, M. \& Ellery Jr., R. (2002). Liquidy constraints and the behavior of the aggregate consumption over the Brazilian business cycle. Estudos Ecônomicos, $32(4): 551-576$.

Campbell, J. (1987). Does saving anticipate declining labor income? An alternative test of the permanent income hypothesis. Econometrica, 55(6):1249-1273.

Cavalcanti, C. B. (1993). Intertemporal substituion in consumption: An empirical investigation for Brazil. Brazilian Review of Econometrics, 13(2):203-229.

Deaton, A. S. (1992). Understanding Consumption. Oxford University Press, New York.

Ellery Jr., R., Gomes, V., \& Sachsida, A. (2002). Business cycle fluctuations in Brazil. Revista Brasileira de Economia, 56(2):269-308.

Engle, R. F. \& Granger, C. W. J. (1987). Co-integration and error correction: Representation, estimation and testing. Econometrica, 55:251-276.

Engle, R. F. \& Kozicki, S. (1993). Testing for common features. Journal of Business and Economics Statistics, 11:369-395.

Engle, R. F. \& Kroner, K. (1993). Multivariate simultaneous generalized ARCH. Discussion Paper 89-57R, July. Department of Economics, University of California, San Diego.

Flavin, M. (1981). The adjustment of consumption to changing expectations about future income. Journal of Political Economy, 89(5). 
Gali, J. (1993). Variability of durable and nondurable consumption: Evidence for six OECD countries. Review of Economics and Statistics, 75:418-428.

Gomes, F. A. R. (2004). Consumo no Brasil: Teoria da renda permanente, formação de hábito e restrição à liquidez. Revista Brasileira de Economia.

Hall, R. (1978). Stochastic implications of the life cycle permanent income hypothesis: Theory and evidence. Journal of Political Economy, 86:971-87.

Issler, J. V. \& Rocha, F. P. (2000). Consumo, restrição a liquidez e bem-estar no Brasil. Economia Aplicada, 4(4):637-665.

Issler, J. V. \& Vahid, F. (2001). Common cycles and the importance of transitory shocks to macroeconomic aggregates. Journal of Monetary Economics, $47(3): 449-475$.

Johansen, S. (1988). Statistical analysis of cointegration vectors. Journal of Economic Dynamics and Control, 12:231-254.

Mankiw, G. (1981). Hall's consumption hypothesis and durable goods. Journal of Monetary Economics, 10:417-25.

Newey, W. \& West, K. (1987). A simple positive semi-definite heteroskedasticity and autocorrelation consistent covariance matrix. Econometrica, 55:703-708.

Ogaki, M. \& Reinhart, C. (1998). Measuring intertemporal substitution: The role of durable goods. Journal of Political Economy, 106(5):1078-98.

Reis, E., Issler, J. V., Blanco, F., \& Carvalho, L. (1998). Renda permanente e poupança precaucional: Evidências empíricas para o Brasil no passado recente. Pesquisa e Planejamento Econômico, 28(2):233-272.

Vahid, F. \& Engle, R. (1997). Codependent cycles. Journal of Econometrics, 80(2):199-121. 


\section{Apêndice A}

\section{A.1 Revisão econométrica}

Considere o modelo de autorregressão vetorial (VAR),

$$
x_{t}=A_{1} x_{t-1}+A_{2} x_{t-2}+\ldots+A_{p} x_{t-p}+\varepsilon_{t},
$$

em que $x_{t}=\left(x_{1 t}, x_{2 t}, \ldots, x_{n t}\right)^{\prime}$ é um vetor $n \times 1$ de variáveis do sistema e $\varepsilon_{t}$ é $n \times 1$, os choques de cada equação, tal que $\varepsilon_{t} \sim \operatorname{iid}(0, \Omega)$. Os componentes do vetor $x_{t}$ serão cointegrados de ordem d, b, $x_{t} \sim C I(d, b)$, se: $i$ ) todos os componentes de $x_{t}$ são $\left.I(d) ; i i\right)$ existe um vetor $\alpha(\neq 0)$ tal que $\alpha^{\prime} x_{t} \sim I(d-b), b>0$. O vetor $\alpha$ é chamado vetor de cointegração (Engle e Granger, 1987).

A partir do modelo VAR podemos definir também os conceitos de ciclo comum e ciclo codependente. Engle e Kroner (1993) introduzem o conceito de ciclo comum através de uma medida de comovimento contemporâneo chamada serial correlation common feature, SCCF. Esta medida verifica a existência de uma combinação linear entre séries estacionárias que seja imprevisível quando se considera o passado histórico destas variáveis. ${ }^{15}$ Vahid e Engle (1997) por sua vez consideram a possibilidade de existir uma combinação linear entre as séries que reduz a dependência do passado, mas não a elimina totalmente, sendo este o conceito de ciclos codependentes. Formalmente, considere o seguinte exemplo:

$$
\begin{aligned}
\left(\begin{array}{l}
y_{1 t} \\
y_{2 t}
\end{array}\right) & =\left(\begin{array}{c}
f_{t} \\
f_{t-1}
\end{array}\right)+\left(\begin{array}{l}
\varepsilon_{1 t} \\
\varepsilon_{2 t}
\end{array}\right) \\
f_{t+1} & =\alpha f_{t}+\theta^{\prime}\left(\begin{array}{l}
\varepsilon_{1 t} \\
\varepsilon_{2 t}
\end{array}\right)
\end{aligned}
$$

em que $\varepsilon_{1 t}$ e $\varepsilon_{2 t}$ são inovações e $y_{1 t}$ e $y_{2 t}$ são variáveis estacionárias. Pode-se observar que,

$$
y_{1 t}-\alpha y_{2 t}=\left(\begin{array}{cc}
1 & -\alpha
\end{array}\right)\left(\begin{array}{c}
\varepsilon_{1 t} \\
\varepsilon_{2 t}
\end{array}\right)+\theta^{\prime}\left(\begin{array}{c}
\varepsilon_{1 t-1} \\
\varepsilon_{2 t-1}
\end{array}\right)
$$

Assim, a combinação linear $y_{1 t}-\alpha y_{2 t}$ só tem correlação com sua primeira defasagem, configurando-se a presença de ciclos codependentes. Por outro lado, se existir uma combinação linear entre as séries estacionárias que produz uma série

\footnotetext{
${ }^{15}$ Note que tais séries devem possuir alguma dependência do passado para que estejamos de fato analisando características comuns.
} 
que é uma inovação, caso em que $\theta=0$, temos a presença de ciclos comuns como definido por Engle e Kozicki (1993).

\section{A.2 Cálculo do consumo de duráveis e não-duráveis}

As Contas Nacionais apresentam o consumo das famílias, sem discriminar o consumo de duráveis e não-duráveis. Além disso, desde 1986 esta série inclui a variação de estoques. De fato, esta série é calculada por resíduo, estando sujeita a todos os tipos de erros de medida.

Para expurgar a variação de estoques, Ellery Jr. et alii (2002) utiliza a publicação anual de matrizes de insumo-produto. Desde 1990 é possível identificar nestas matrizes a variação de estoque, para todos os setores, de 1990 a 1998. Para os anos de 1987, 1988 e 1989 esse autores utilizam uma interpolação linear.

Para dissociar o consumo de duráveis e não-duráveis, Ellery Jr. et alii (2002) notam que a participação do consumo de não-duráveis nos anos de 1970 e 1980 , extraídas das respectivas matrizes de insumo-produto, apresenta uma pequena alteração de 0.62 para 0.61, o que, segundo eles dá evidente suporte para uma hipótese de estabilidade. Daí, é possível usar uma ferramenta de interpolação a partir das participações do consumo de não-duráveis disponíveis nas matrizes de insumo-produto de 1970, 1975, 1980, 1985 e 1990. Enfim, esses autores empregam a seguinte metodologia:

1. Interpolação linear simples para completar a série de participação de consumo de duráveis;

2. Geração de uma série de choques com média zero e variância igual à série observada de 1990 a 1998 ;

3. Participação estimada do consumo de duráveis é igual a soma da interpolação linear simples e do choque;

4. Multiplicação da participação estimada pela série de consumo total;

5. Obtenção da série de consumo de não-duráveis por resíduo.

Com isso é obtida a série de consumo de não-duráveis para o período de 1970 a 1990. De 1990 em diante, é utilizada as matrizes anuais de insumo-produto para obter a participação de consumo de não-duráveis. 


\section{A.3 Cálculo do estoque inicial de bens de consumo duráveis}

Considere a lei de movimento do estoque de bens de consumo duráveis:

$$
k_{t}=c d_{t-1}+(1-\delta) k_{t-1}
$$

Daí,

$$
k_{t}=(1-\delta)^{t} k_{0}+\sum_{j=1}^{t}(1-\delta)^{j-1} c d_{t-j}
$$

Através desta lei de movimento é possível obter ainda:

$$
\begin{aligned}
k_{0} & =c d_{-1}+(1-\delta)\left[c d_{-2}+(1-\delta) k_{-2}\right] \\
& =(1-\delta)^{T} k_{-T}+\sum_{j=1}^{T}(1-\delta)^{j-1} c d_{-j}
\end{aligned}
$$

Supondo que o gasto em bens duráveis tenha evoluído à taxa $g$ até o instante zero, isto é, $c d_{-j}=c d_{0}(1+g)^{-j}$, isto implica que a equação (A.3.3) torna-se:

$$
k_{0}=(1-\delta)^{T} k_{-T}+\left(\frac{c d_{0}}{1+g}\right) \sum_{j=0}^{T-1}\left(\frac{1-\delta}{1+g}\right)^{j}
$$

Note que $1-\delta<1+g$, logo tomando o limite da equação (A.3.4) obtemos:

$$
k_{0}=\frac{c d_{0}}{g+\delta}
$$

Portanto, obtemos $k_{0}$ como função de $i_{0}, g$ e $\delta$. Apesar de possuir o valor $i_{0}$, é preciso obter um valor que seja representativo do processo do gasto em duráveis de $-\infty$ a 0 . Para tanto considere a seguinte estimação da taxa de crescimento média para dados discretos. No modelo contínuo temos, $c d_{t}=c d_{0} e^{g t}$, em que $g$ é taxa de crescimento. Para estimar $g$ a partir de um modelo de regressão linear fazemos $\ln \left(c d_{t}\right)=\ln \left(c d_{0}\right)+g t$. Assim, ao estimar o valor da taxa de crescimento do caso discreto, $g_{d}$, fazemos:

$$
g_{d}=\frac{c d_{t}-c d_{t-1}}{c d_{t-1}}=\frac{e^{g t}-e^{g(t-1)}}{e^{g(t-1)}}=e^{g}-1
$$

Definimos então o gasto em bens duráveis estacionário: 


$$
c d_{t}^{\text {Estacionário }} \equiv \frac{c d_{t}}{\left(1+g_{d}\right)^{t}}
$$

Assim o gasto inicial é dado pela média do gasto estacionário:

$$
c d_{0}=\frac{1}{T} \sum_{j=1}^{T} i_{t}^{\text {Estacionário }}
$$

Utilizando as equações (A.3.5) e (A.3.6) encontramos $k_{0}$ como função de $g$ e $\delta$. Determinando então estes dois parâmetros e utilizando a equação (A.3.2) obtemos toda série de estoque de capital. Para tanto considere o seguinte:

$$
\begin{array}{r}
k_{t}=\int_{-\infty}^{t} e^{\delta(\tau-t)} c d_{\tau} d \tau \\
=\int_{-\infty}^{t} e^{\delta(\tau-t)}\left(e^{g(\tau-t)} c d_{t}\right) d \tau \\
=\frac{c d_{t}}{(\delta+g)}
\end{array}
$$

Assim dividindo ambos os lados da equação (A.3.7) por y obtemos:

$$
\frac{k_{t}}{y_{t}}=\frac{1}{(\delta+g)} \frac{c d_{t}}{y_{t}}
$$

Como $g$ é taxa de crescimento do gasto em duráveis, conhecendo $\delta$ é obtido o capital inicial. Os dados indicam que $g=0.033$. Utilizamos então 5 valores distintos para $\delta$, a saber a saber $1.67 \%, 3.67 \%, 6.67 \%, 8.67 \%$ e $11.67 \%$. 